\title{
Should we stop using the term "malformation" for Chiari type I?
}

\section{Concezio Di Rocco ${ }^{1}$}

Published online: 7 August 2019

(C) Springer-Verlag GmbH Germany, part of Springer Nature 2019

Very few pathological conditions have stimulated the interest of the pediatric neurosurgeon as the so-called Chiari type I malformation (CMI) in spite of the uncertainty of its definition, diagnosis, treatment modalities, and surgical outcomes.

The term "malformation" implies a congenital origin although the caudal descent of the hindbrain structures was conceived by Hans von Chiari in his first description as the mechanical effect and the pressure gradient exerted by concomitant hydrocephalus. He actually did not refer to symptoms or signs of cerebellar or brainstem dysfunction. It is possible that the term malformation was just due to the zeal of E. Schwalbe and M. Gredig, two students of J. Arnold. In fact, they associated the name of their professor to that of Chiari in describing a case of spinal dysraphism and emphasized the connection between the herniation of the spinal cord and the deformity of the posterior cranial fossa, using the eponym: "Arnoldsche und Chiarische Missbildung." Indeed, Julius Arnold had reported the pathological findings of an infant dying shortly after delivery. The child had a large herniation of the thoraco-lumbar spinal cord and the brainstem was described as underdeveloped with a downward draw of some of the lower parts of the cerebellum and the fourth ventricle in the spinal canal. Arnold however had not been able to explain the deformity of the brainstem. The paper of Schwalbe and Gredig had an extremely high impact and the eponym they coined was diffusely utilized to indicate the posterior cranial fossa and hindbrain anomalies associated with myelomeningocele, namely the descent of the cerebellar tonsils and eventually the inferior vermis and the fourth ventricle into the upper spinal canal. As a matter of fact, the eponym extended the concept of malformation to the other congenital abnormalities of the posterior cranial fossa described by Chiari characterized in his classification in four degrees of

Concezio Di Rocco

dirocco@ini-Hannover.de

1 Pediatric Neurosurgery, International Neuroscience Institute, Hannover, Germany severity, including the Chiari type I identified as the simple caudal herniation of the cerebellar tonsils into the spinal canal.

The current wide availability of modern diagnostic neuroimaging study has led to the identification of the caudal descent of the cerebellar tonsils below the rim of the foramen magnum in approximately $1 \%$ of children undergoing a magnetic resonance investigation to identify possible cerebral abnormalities in cases of aspecific psycho-motor retardation, seizure disorders, chronic headache, or after head injury. Several prospective observational studies in such a type of population allowed to conclude that in almost all the cases, the condition remains stable in control exams in the subsequent years without any clinical manifestation. In some children, a spontaneous regression of the cerebellar tonsils within the cranial cavity is even observed. The same evolution is reported in adults too, obviously challenging its malformative nature. To call "malformation" such a type of phenomenonwithout any doubts a possible normal variant-has important practical implications. First, for the family that could be convinced that their son/daughter is affected by a congenital disorder which requires continuous surveillance as well as limitations in daily physical activities. The term "malformation" could also cause parents' anxiety for its possible inheritance. Furthermore, the acceptance of a prophylactic surgical correction, even weighted by possible complications, would be favored resulting in an excess of improper or useless surgical procedures. For the neurosurgeon, the increasing number of incidentally recognized caudal displacement of the cerebellar tonsils on MRI studies is also a cause of obvious anxiety when faced with the family's request for a prognosis, further dilucidation about the cause of the "malformation," the best management, and, when surgical treatment is suggested, its possible complications and its goals. Unfortunately, the very few reported cases of abrupt neurological deterioration and even death following head injury weight more than the thousands of subjects that conduct normal life harboring the caudal descent of the cerebellar tonsils. Anyway, to eliminate the term "malformation," in my opinion, would favor a more appropriate information as well as a better understanding of the 
condition and a better acceptance of its very low associated risk in untreated children by the family.

About half of the pediatric population with the so-called $\mathrm{CMI}$ is asymptomatic at diagnosis. In the remaining half, the clinical manifestations are so numerous and heterogeneous that they can be hardly considered specific of the "malformation." Actually, the "malformation" can be found and in several instances seen to progress in a malformative context so that it is logical to consider the alternative hypothesis that the CMI is the result rather than the cause of the condition. Indeed, the CMI can be found in the evolutive phases of children with anomalies of the skull base, hypoplasia of the posterior cranial fossa, single and complex craniosynostoses, bone metabolism abnormalities, CSF dynamic disorders, cranial volume reductions resulting from chronic CSF depletion either spontaneous or iatrogenic (e.g., repeated CSF spinal subtractions, action of an extrathecal CSF shunting), and syndromes leading to abnormal cerebellum growth. All of them may constitute the causative malformative context accounting for the development of the "malformation." In a relevant proportion of the cases, the CMI is only the result of an acquired cranio-cerebral disproportion that, besides the caudal descent of the cerebellar tonsils, is responsible also for a cranial progressive ascend of the superior cerebellar vermis into the cistern of the great vein of Galen. To restore normal craniocerebral spatial relationships almost ever results in the disappearance of the "malformation." In other terms, the presence of the cerebellar tonsils within the upper spinal canal is a functional state rather than a malformation which can be properly corrected when its causes are recognized adequately.

In front of this impressive variety of etiologies and pathophysiogenetic mechanisms, the only identifying finding of the malformation is the 3-4- to 5-mm descent of the cerebellar tonsils below the posterior ridge of the foramen magnum! The level of the descent is often utilized as an indicator of the severity of CMI or as a classification criterion. In a similar way, the tonsillar rising after the operation is regarded as the main indicator for its success. The limitations of such an approach are obvious and account for the difficulties experienced in establishing the surgical indication, in the choice of the surgical modalities and, even more, in the evaluation of the surgical outcome. Almost all the available surgical reports concern series of patients accomunated by the "caudal descent" of the cerebellar tonsils - malformation of Chiari type I-without any mention of the causative malformative context that by itself is the most important factor conditioning the management of the condition and the rate of the surgical success or failure. It is quite possible that CMI operated malformations in the large published series comprise also ignored or still unknown genetic disorders of the bone metabolism or cerebellar growth that bear their own prognosis not necessarily responding to a surgical procedure. Taking into account the just-mentioned consideration, the current discussion on the best surgical approaches and the evaluation of the outcomes appear quite unreliable, preventing any significant comparison of the experiences and impairing the actual progress in the management of the condition.

It is time, in my opinion, to contextualize CMI in order to manage subjects harboring a caudal (and, in some case, cranial) dislocation of the cerebellar tonsils adequately. Only the recognition of the causes of the phenomenon may allow to distinguish the numerous patients with acquired CMI from the few cases of real congenital malformation of the hindbrain for which only the eponym of Chiari malformation type I should be preserved. For all the other cases, a distinction into specific subgroups is becoming necessary in order to select the appropriate management and compare the surgical outcomes. Indeed, it is not logical to compare the utilized surgical modalities and the obtained result if patients of CMI depending on a platybasia or a hypoplasic posterior cranial fossa are reported under the umbrella definition of Chiari malformation type I. Actually, the same surgical procedure, posterior fossa decompression, with or without dural expansion, might result in a further hindbrain descent in the first case or in "ascent" of the cerebellar tonsils in the second case. I hope that this Special Annual Issue of CNS, organized so well by Drs. Massimi and Thompson, may constitute an important base to proceed in this direction.

As a final suggestion, let me invite you considering the opportunity of a new "contextualized" classification that the ISPN could elaborate by distinguishing the different conditions leading to the so-called Chiari type I malformation. For example, besides eliminating the term "malformation," we could maintain, for historical reasons, the name of Chiari adding letters that could identify specific subgroups according to the most probable causative context, e.g., Chiari-CS (associated to craniosynostosis), Chiari-P (associated to platybasia), Chiari-CCD (associated to cranio-cerebral disproportion), Chiari-SP (associated to secondary neurulation abnormalities), Chiari-M (associated to bone metabolic disorders), and so on. In such a way, the indication for the surgical corrections could be based on more solid elements and the surgical modalities designed more appropriately. Even more important, we could rely on a better understanding and prevention of the surgical complications as well as on a more reliable evaluation of the surgical outcomes.

Publisher's note Springer Nature remains neutral with regard to jurisdictional claims in published maps and institutional affiliations. 